\title{
Long-term dynamics of Norovirus transmission in Japan, 2005-2019
}

\author{
Megumi Misumi $^{1,2}$, Hiroshi Nishiura ${ }^{\text {Corresp. 1,3 }}$ \\ ${ }^{1}$ Graduate School of Medicine, Hokkaido University, Sapporo, Hokkaido, Japan \\ 2 Rumoi City Hospital, Rumoi city, Hokkaido, Japan \\ ${ }^{3}$ School of Public Health, Kyoto University, Kyoto city, Kyoto, Japan \\ Corresponding Author: Hiroshi Nishiura \\ Email address: nishiurah@gmail.com
}

Norovirus continues to evolve, adjusting its pathogenesis and transmissibility. In the present study, we systematically collected datasets on Norovirus outbreaks in Japan from 2005 to 2019 and analyzed time-dependent changes in the asymptomatic ratio, the probability of virus detection, and the probability of infection given exposure. Reports of 1728 outbreaks were published, and feces from all involved individuals, including those with asymptomatic infection, were tested for virus in 434 outbreaks. We found that the outbreak size did not markedly change over this period, but the variance in outbreak size increased during the winter (November-April). Assuming that natural history parameters did not vary over time, the asymptomatic ratio, the probability of virus detection, and the probability of infection given exposure were estimated to be $18.6 \%, 63.3 \%$, and $84.5 \%$, respectively. However, a model with time-varying natural history parameters yielded better goodness-of-fit and suggested that the asymptomatic ratio varied by year. The asymptomatic ratio was as high as $25.8 \%$ for outbreaks caused by genotype GIl.4 noroviruses. We conclude that Norovirus transmissibility has not changed markedly since 2005 , and that yearly variation in the asymptomatic ratio could potentially be explained by the circulating dominant genotype. 


\section{TITLE}

2 Long-term dynamics of Norovirus transmission in Japan, 2005-2019

3

4 Authors:

5 Megumi Misumi ${ }^{1,2}$ and Hiroshi Nishiura ${ }^{1,3 *}$

6

\section{Affiliations:}

$8{ }^{1}$ Graduate School of Medicine, Hokkaido University, Kita 15 Jo Nishi 7 Chome, Kita-ku,

9 Sapporo-shi, Hokkaido 060-8638, Japan

10 2Rumoi City Hospital, Shinonome 2-16-1, Rumoi City, Hokkaido 077-8511, Japan

$11{ }^{3}$ Kyoto University School of Public Health, Yoshidakonoecho, Sakyoku, Kyoto 606-8501, Japan

$13 *$ Correspondence to:

14 Hiroshi Nishiura

15 Kyoto University School of Public Health

16 Yoshidakonoecho, Sakyoku, Kyoto 6068501, Japan

17 Tel: +8175753 4456, Fax: +81757534458

18 E-mail: nishiurah@gmail.com 
19 Abstract

20 Norovirus continues to evolve, adjusting its pathogenesis and transmissibility. In the present

21 study, we systematically collected datasets on Norovirus outbreaks in Japan from 2005 to 2019

22 and analyzed time-dependent changes in the asymptomatic ratio, the probability of virus

23 detection, and the probability of infection given exposure. Reports of 1728 outbreaks were

24 published, and feces from all involved individuals, including those with asymptomatic infection,

25 were tested for virus in 434 outbreaks. We found that the outbreak size did not markedly change

26 over this period, but the variance in outbreak size increased during the winter (November-April).

27 Assuming that natural history parameters did not vary over time, the asymptomatic ratio, the

28 probability of virus detection, and the probability of infection given exposure were estimated to

29 be $18.6 \%, 63.3 \%$, and $84.5 \%$, respectively. However, a model with time-varying natural history

30 parameters yielded better goodness-of-fit and suggested that the asymptomatic ratio varied by

31 year. The asymptomatic ratio was as high as $25.8 \%$ for outbreaks caused by genotype GII.4

32 noroviruses. We conclude that Norovirus transmissibility has not changed markedly since 2005,

33 and that yearly variation in the asymptomatic ratio could potentially be explained by the

34 circulating dominant genotype.

35 Keywords

36 Caliciviridae; Natural history; Transmission; Asymptomatic ratio; Epidemiology; Epidemic 
Norovirus is a genus of RNA viruses in the family Caliciviridae and consists of 10 genogroups (GI-GX), among which GI, GII, and GIV infect humans (de Graaf, van Beek \& Koopmans, 2016; Chhabra et al., 2019). At present, GI and GII are the most frequently detected genogroups; in particular, GII.4, a genotype of the GII genogroup, continues to be most prevalent. GII.4 noroviruses evolve antigenically, producing a new variant every $2-4$ years (Lindesmith et al., 2008; White, 2014). Norovirus infection is the leading cause of acute gastroenteritis worldwide (Lopman et al., 2012; Pringle et al., 2015; O’Ryan, Riera-Montes \& Lopman, 2017) and is responsible for substantial morbidity, mortality, and healthcare-associated costs (Hall et al., 2013; Ahmed et al., 2014; Gaythorpe et al., 2018; Chhabra et al., 2019). Transmission is highly seasonal and most intense in the middle of winter (Phillips et al., 2010; Hall et al., 2013; Ahmed et al., 2014; Rushton et al., 2019). With improved efficiency and lower costs of the reverse transcription polymerase chain reaction (RT-PCR) technique, Norovirus infection is now known to be responsible for both sporadic infections and epidemics (Pringle et al., 2015).

Norovirus continues to evolve, modifying its pathogenesis and transmissibility (White, 2014) to ensure its continued prevalence in human populations. The major routes of transmission are via contaminated foods and the fecal-oral route (Rushton et al., 2019), although vomit from symptomatic patients also aerosolizes millions of virions. The minimum number of virions required to establish an infection is as low as 18 particles (Teunis et al., 2008). Asymptomatic carriers of Norovirus complicate the control of outbreaks (Phattanawiboon et al., 2020); the duration of viral shedding in feces and the dose of virus excreted are similar in asymptomatic carriers and symptomatic patients (Teunis et al., 2015; Newman et al., 2016). This phenomenon, especially among asymptomatic food handlers, has caused critical difficulties in controlling 
61 outbreaks (Ozawa et al., 2007; Barrabeig et al., 2010; Nicolay et al., 2011; Jeong et al., 2013;

62 Franck et al., 2015; Sabria et al., 2016; Chen et al., 2016; Qi et al., 2017).

Norovirus genotype GII.4 is widely prevalent worldwide (Ramani, Atmar \& Estes, 2014;

64 Kambhampati, Koopmans \& Lopman, 2015). Japan is no exception, having continuously detected the genotype since 2006 (Motomura et al., 2010). Genotype GII.4 does not necessarily induce symptomatic illness in infected individuals (Ozawa et al., 2007; Miura, Matsuyama \&

67 Nishiura, 2018), increasing the possibility of asymptomatic transmission. In recent decades, this genotype has been detected most frequently, underscoring the difficulty in controlling its transmission (Miura, Matsuyama \& Nishiura, 2018). The dominance of GII.4 implies that its transmissibility has increased over time (Matsuyama, Miura \& Nishiura, 2017), potentially increasing outbreak sizes. Since 2007, the Ministry of Health, Labour and Welfare of Japan has issued revised guidance for food handling facilities to prevent large-scale outbreaks. The guidelines recommend routine testing of fecal samples for noroviruses among asymptomatic food handlers from October until March. noroviruses and to clarify the importance of testing fecal samples of asymptomatic food handlers as part of prevention activities. To achieve the goal, we systematically collected datasets on

78 Norovirus outbreaks documented by the prefectural institutes of public health across Japan from 2005 to 2019. In some of these datasets, both symptomatic patients and asymptomatic individuals underwent thorough laboratory-based fecal testing. We analyzed time-dependent

81 changes in the asymptomatic ratio, the probability of virus detection, and the probability of

82 infection given exposure in this long-term series. 
83

84

85

86

87

\section{Materials \& Methods}

\section{Data sources: Epidemiological records of outbreaks}

Japan consists of 47 prefectures and each prefecture has an institute of public health responsible for the laboratory testing of notifiable infectious diseases. In addition to these prefectural institutes, major urban cities (e.g., prefectural capitals) have institutes of public health; there are 83 such institutes in total throughout Japan. Whenever an outbreak of norovirus occurs, laboratory samples are analyzed and the results recorded. Once the occurrence of cases is recognized, the outbreak is detected via notifications from physicians to local health centers or through reporting by non-physicians or interviews by healthcare workers. Notification is required by Food Hygiene Law (Ministry of Health, Labour and Welfare, 2019). Fecal sampling of all asymptomatic and symptomatic individuals takes place when two or more cases arise from a food handling facility, as food poisoning is suspected. Confirmatory diagnoses are made by RTPCR, regardless of symptoms. Feces from all individuals, including customers and food handlers, are sampled. A 10\% emulsion is prepared and centrifuged at 10,000 rpm for 20 minutes in a refrigerated centrifuge, then RNA is purified from the supernatant (Ministry of Health, Labour and Welfare, 2003). Noroviruses are screened for genogroups GI and GII, and a subset of samples are further examined for genotyping.

The annual reports of 78 of the 83 institutes of health were screened from 2005 to 2019 (the remaining institutes do not widely publish their annual reports). In this study, we investigated the records from all these annual reports, and systematically collected datasets on the locations (prefecture) of norovirus outbreaks, the months and years of occurrence, the numbers of symptomatic cases, the numbers of exposed individuals, the numbers of laboratory 
105 tests performed and their results for symptomatic individuals, and the numbers of laboratory tests

106 performed and their results for asymptomatic individuals (e.g., asymptomatic food handlers).

\section{Descriptive analysis of outbreaks}

108 We investigated the descriptive features of the outbreaks identified, including the geographic 109 distribution, temporal distribution (by year), seasonality (measured as monthly incidence), and

110 the distribution of facilities where the outbreaks occurred. Age was not consistently reported

111 across outbreaks, so was excluded from the analysis. Asymptomatic cases were defined as

112 individuals with complete absence of symptoms throughout the course of infection.

113 We then collected datasets on outbreak sizes over time to identify any changes in virus

114 transmissibility and outbreak seasonality. To examine trends in outbreak size over time, two

115 independent analyses were conducted. First, to investigate the impact of seasonal variation on

116 virus transmission, the outbreak sizes from November to February (i.e., winter) were compared

117 with those of outbreaks during other months. Second, yearly variations in outbreak size were

118 investigated over time.

\section{Estimation of the asymptomatic ratio}

120 In addition to descriptive analyses, we used a statistical model to estimate the asymptomatic ratio

$121(s)$ and other parameters including the probability that infected individuals avoided symptomatic

122 illness $(q)$ and the probability of virus detection in an infected individual $(p)$, as proposed

123 elsewhere (Miura, Matsuyama \& Nishiura, 2018). While detailed mathematical descriptions can

124 be found elsewhere (Miura, Matsuyama \& Nishiura, 2018), it should be emphasized that testing

125 fecal samples of all individuals involved in each outbreak allowed us to avoid ascertainment

126 bias. In short, the model considered three pieces of information: the infection process, illness 
127 onset, and viral shedding. Assuming that all three parameters did not vary over time, and given

128 the input values for the number of symptomatic individuals $\left(n_{\mathrm{i}}\right)$, the number of asymptomatic

129 individuals $\left(m_{\mathrm{i}}\right)$, the number of virus-positive asymptomatic individuals $\left(y_{\mathrm{i}}\right)$, and viral shedding

130 by symptomatic individuals $\left(x_{\mathrm{i}}\right)$ in each outbreak $i$, the total likelihood $L$ for estimating

131 parameters was computed as:

$$
L(p, q, s ; \text { data })=L_{1} L_{2} L_{3}
$$

132 where

$$
\left\{\begin{array}{c}
L_{1}(p, s ; \mathbf{n}, \mathbf{m})=\prod_{i}\left(\begin{array}{c}
n_{i}+m_{i} \\
n_{i}
\end{array}\right)(p(1-s))^{n_{i}}(1-p(1-s))^{m_{i}}, \\
\left.L_{2}(p, q, s ; \mathbf{m}, \mathbf{y})=\prod_{i}\left(\begin{array}{c}
m_{i} \\
y_{i}
\end{array}\right)\left(\frac{p s q}{1-p+p s}\right)\right)^{y_{i}}\left(1-\left(\frac{p s q}{1-p+p s}\right)\right)^{m_{i}-y_{i}} \\
L_{3}(q ; \mathbf{n}, \mathbf{x})=\prod_{i}\left(\begin{array}{c}
n_{i} \\
x_{i}
\end{array}\right) q^{x}(1-q)^{\left(n_{i}-x_{i}\right)} .
\end{array}\right.
$$

133 That is, each data generation process was assumed to be described by binomial sampling. We

134 obtained maximum likelihood estimates of $p, q$, and $s$ by minimizing the negative logarithm of

135 equation (1), and the 95\% confidence intervals (CIs) were computed using the profile likelihood.

136 Alternatively, we used a model in which unknown parameters varied every single year because

137 the dominant genotype (e.g., GII.4) varied over time. To identify any improved fit despite the

138 increased number of parameters, Akaike's information criterion (AIC) was computed and

139 compared across the different models.

Data sharing statement

141 The original outbreak size data are available as Online Supporting Material. 


\section{Ethical considerations}

143 The present study was based exclusively on published data. Therefore, it did not require ethical 144 approval.

\section{Results}

146 In total, the annual reports contained records for 1728 norovirus outbreaks. Of these, 434

147 outbreaks had full datasets, including numbers of exposed asymptomatic individuals. Figure 1A

148 shows the geographic distribution of cases from April 2005 to March 2019 in different

149 geographic regions. Of the 1728 outbreaks, the Kinki region, containing the third most populated 150 prefecture in Japan (Osaka), had the most outbreaks (605, 35.0\%). The second largest outbreak

151 count was in the Tohoku region, located in northeastern Japan, with 393 outbreaks (22.7\%).

152 Hokkaido had only 27 documented outbreaks (1.5\%). The yearly number of outbreaks was 153 highest in 2006, with 211 outbreaks, followed by 2010 with 171 outbreaks (Figure 1B). The 154 mean number of outbreaks was 118 per year. Seasonally, the highest frequency was seen in 155 December (386 outbreaks), followed by January (311 outbreaks) and February (227 outbreaks)

156 (Figure 1C). The lowest frequency of outbreaks was observed in September (15 outbreaks).

157 Figure 1D and 1E show the outbreak settings (facilities) in Nara and Tokyo by year. In Nara, 158 approximately $70 \%$ of outbreaks occurred in nursery or elementary schools. In Tokyo, nursery 159 schools and elderly-care facilities dominated the outbreak settings. There was no clear pattern of 160 change in outbreak settings over time.

161 Of the 434 outbreaks in which asymptomatic individuals were tested, the most frequent 162 locations were restaurants and food courts (203 outbreaks), followed by food-handling facilities 163 (59 outbreaks), Japanese ryokan (lodgings; 33 outbreaks), and hotels (27 outbreaks). The sizes of 
164 confirmed outbreaks ranged from 2 to 308 cases, with a median of 16 cases (lower and upper 165 quartiles: 9 and 30 cases, respectively). The mean number of symptomatic virus-positive cases

166 per outbreak was 6.7 (standard deviation [SD], 5.3 individuals). The mean number of exposed 167 asymptomatic food handlers was 7.5 individuals (SD, 7.2 individuals). The median number of

168

169

170

171

172

173

174

175

176

177

178

179

180

181

182

183

184

185

186

asymptomatic virus-positive food handlers was 1 individual (range, 0-16 individuals).

Figure 2A compares outbreak size by season. Whereas the median (range) outbreak size was 15.5 (2-99) cases in May-October, the range of outbreak sizes expanded from November to April, with a median of 16.0 cases (range, 2-308 cases). The SD of outbreak size in summer was 20.5 individuals compared with 33.1 individuals in winter; the variance was significantly greater in winter according to an F-test $(\mathrm{p}<0.0001)$. Figure $2 \mathrm{~B}$ examines time-dependent variation in outbreak size. Overall, outbreak size remained stable over these years, with mean outbreak sizes of $5-15$ cases.

Assuming that the asymptomatic ratio and other natural history parameters did not vary over time, the asymptomatic ratio (parameter $s$ ) was estimated as $18.6 \%$ (95\% CI: $17.4 \%-$ $19.9 \%)$, and the risks of infection and virus positivity were $63.3 \%(95 \% \mathrm{CI}: 62.1 \%-64.5 \%)$ and $84.5 \%$ (95\% CI: $88.3 \%-85.7 \%)$, respectively. Table 1 shows estimates of the natural history parameters that were assumed to change as a function of the year of outbreak. Overall, the estimates of the risk of infection and virus positivity did not show clearly increasing or decreasing trends, and the arithmetic averages for the asymptomatic ratio, risk of infection, and risk of virus positivity were $18.6 \%, 63.9 \%$, and $85.4 \%$, respectively. However, we found that the asymptomatic ratio varied over time: it was smallest at 4.4\% (95\% CI: $1.4 \%-9.9 \%)$ in 2005 , and largest at $25.5 \%(95 \%$ CI: $21.3 \%-30.1 \%)$ in 2007 . The AIC of the model in which the natural history parameters remained constant over time was 22,292, whereas that of the model with 
187 time-varying parameters was 15,462 , indicating that the model including yearly variations better 188 captured the observed data.

Table 2 shows the natural history parameters by genogroup. The risks of infection and

190

191

192

193

194 195

196

197

198

199

200

201

202

203

204

205

206

207

208

209

viral shedding were similar across genogroups. However, the asymptomatic ratio of GII.4

genotype outbreaks was as high as 25.8 (95\% CI: $22.0 \%-29.9 \%)$. This estimate was higher than

the asymptomatic ratio of GI group outbreaks alone or GII group outbreaks alone when genotyped GII.4 was excluded, but these differences were not statistically significant. More detailed comparisons of genogroup/genotype data are available as Online Supplementary Table 1.

\section{Discussion}

In this study, we investigated the long-term dynamics of norovirus outbreaks in Japan from 2005 to 2019 by systematically analyzing reports of 1728 outbreaks published by the prefectural institutes of public health and examining the temporal and spatial distributions of the outbreaks. We showed that outbreak size did not vary markedly over the years, but the variance in outbreak size increased during winter (November-April). Assuming that natural history parameters did not vary with time, the asymptomatic ratio, the probability of virus detection, and the probability of infection given exposure were estimated to be $18.6 \%, 63.3 \%$, and $84.5 \%$, respectively. The model with time-varying parameters yielded a smaller AIC value, and suggested that the asymptomatic ratio varied by year. The asymptomatic ratio for infection with genotype GII.4 was as high as $25.8 \%$.

In this study, we demonstrated that outbreak size did not vary and that the natural history parameters governing the risk of infection and the risk of a virus-positive outcome remained stable from 2005 to 2019, indicating that there has been no apparent trend or increase in the 
210 transmissibility of Norovirus over this period. The absence of marked increases in these

211 parameters, as was seen following the 2003/04 season by Matsuyama et al. (2017), suggested

212 that an increase in the infectiousness of the virus occurred between 2003 and 2005, shortly

213 before the period covered by our study. Transmissibility was considered to have peaked in

214 2006/07 by Matsuyama et al. (2017). We did not identify a similar trend in the present study,

215 potentially in part because of the limited number of samples from 2005 and earlier. However,

216 yearly variations in the asymptomatic ratio were identified, potentially reflecting the dominant

217 genotype circulating in the corresponding winter; an increase in the asymptomatic ratio was

218 particularly noticeable in 2007. However, genotyping was not complete for all genogrouped

219 samples, and other published studies have suggested that the asymptomatic ratios of GII.4 and

220 other genotypes were overall comparable (Ballard et al., 2015; Bucardo et al., 2017; Saito et al.,

221 2014; Colston et al., 2019; Bhavanam et al., 2020). Although no long-term trends were

222 identified in our study, marked seasonal variations were observed. Dey et al. (2010) sampled

223 Noroviruses between 1995 and 2007 across seven different geographic areas of Japan and

224 showed that the annual peaks always occurred between November and January. Our findings

225 echo those of Dey et al. (2010); the increased variance in outbreak size during winter (without a

226 corresponding increase in the mean outbreak size) indicates that seasonal preference was not

227 related to increased transmissibility but rather an increased number of outbreaks in winter

228 season.

229 The large asymptomatic ratio estimated for the GII.4 genotype is consistent with the

230 results of a prior study by Miura et al. (2018) in Japan. However, clinical surveillance studies

231 have indicated that GII.4 likely caused more symptomatic infections than other genotypes

232 (Kazama et al., 2016; Fumian et al., 2019). Miura et al. (2018) estimated the asymptomatic ratio 
233 to be high as $32.1 \%$ in 2006 , whereas our estimate during the years examined was $25.8 \%$.

234 Modeling studies can avoid the ascertainment biases of laboratory testing and genotyping and

235 allow explicit statistical estimation of the asymptomatic ratio. The basis for inconsistencies

236 between modelling and surveillance studies should be further explored by comparing detailed

237 clinical symptoms. Our goal here was to study the long-term dynamics of Norovirus outbreaks,

238 whereas it was evident from the results of Miura et al. (2018) that the year 2006 was clearly

239 dominated by GII.4 (Dey et al., 2010; Matusyama et al., 2017). Therefore, we report here that the

240 asymptomatic ratio varies by year, whereas the transmissibility of the virus in the last decade has

241 shown no clear trend. Unfortunately, the causal impact of GII.4 in increasing transmissibility

242 could not be investigated in the present study. In the literature, the dominance of GII.4 has not

243 been attributed to transmissibility but rather to epochal evolution and continuous changes in

244 antigenicity (Debbink et al. 2012; Parra et al. 2017; Tohma et al. 2019). Our results showed that

245 there were no marked changes in the composition of genogroups/genotypes during the period of

246 our analysis.

247 This study had three technical limitations. First, reported outbreaks must have been

248 greater in size than sporadic occurrences, so our study samples may have been affected by

249 reporting bias. For this reason, Norovirus transmissibility could have been overestimated. In fact,

250 Qi et al. (2018) estimated that the asymptomatic ratio based on 15 outbreak datasets was high.

251 Second, our estimates are predominantly based on the choice of food handlers as the control

252 group, who may be more exposed to the virus than other individuals. If so, the transmissibility of

253 Norovirus could have been overestimated because of sampling bias. Similarly, larger outbreaks

254 would have been sampled more frequently, again elevating estimates of transmissibility. Third,

255 our model involved simplistic assumptions (e.g., viral shedding from food handlers). Norovirus 
256 can be detected in feces for up to 4 weeks after infection (Rockx et al., 2002; Kirkwood \&

257 Streitberg, 2008), and virus excretion occurs even during the recovery period (Bucardo, 2018).

258 Because asymptomatic hosts were all sampled among food handlers, the possibility that

259 individuals within the recovery period were included cannot be ignored.

260 Although further studies are required, the present study was based on systematically

261 collected outbreak data from 2005 to 2019 , with a sample size of 1728 outbreaks across Japan. In

262 conclusion, our data show that the transmissibility of Norovirus has not changed over time.

263 However, yearly variation in the asymptomatic ratio suggested a potentially higher asymptomatic

264 ratio for GII.4 compared with other genotypes. This possibility should be further verified in 265 future studies.

\section{Funding}

268 H.N. received funding from Health and Labor Sciences Research Grants (19HA1003, 269 20CA2024, and 20HA2007), the Japan Agency for Medical Research and Development 270 (JP19fk0108104, JP20fk0108140 and JP20fk0108535s0101), the Japan Society for the 271 Promotion of Science KAKENHI (17H04701 and 21H03198), the Inamori Foundation, GAP

272 Fund Program of Kyoto University, the Japan Science and Technology Agency CREST program 273 (JPMJCR1413); and the SICORP (e-ASIA) program (JPMJSC20U3). This study was also

274 supported by German Federal Ministry of Health (BMG) COVID-19 Research and Development 275 funding to the World Health Organization. The funders played no role in the study design, data 276 collection and analysis, decision to publish, or preparation of the manuscript.

\section{Acknowledgments}


278 We thank Janine Miller, PhD, from Edanz Group (https://en-author-services.edanz.com/ac) for 279 editing a draft of this manuscript.

\section{References}

282

283

284

285

286

287

288

289

290

291

292

293

294

295

296

297
Ahmed SM, Hall AJ, Robinson AE, Verhoef L, Premkumar P, Parashar UD, Koopmans M, Lopman BA. 2014. Global prevalence of norovirus in cases of gastroenteritis: A systematic review and meta-analysis. The Lancet Infectious Diseases 14:725-730 DOI: 10.1016/S14733099(14)70767-4.

Barrabeig I, Rovira A, Buesa J, Bartolomé R, Pintó R, Prellezo H, Domínguez A. 2010.

Foodborne norovirus outbreak: The role of an asymptomatic food handler. BMC Infectious Diseases 10:269 DOI: 10.1186/1471-2334-10-269.

Bhavanam S, Freedman SB, Lee BE, Zhuo R, Qiu Y, Chui L, Xie J, Ali S, Vanderkooi OG, Pang XL, On Behalf Of The Alberta Provincial Pediatric Enteric Infection Team Appetite. 2020 Differences in illness severity among circulating norovirus genotypes in a large pediatric cohort with acute gastroenteritis. Microorganisms 8:1873 DOI: 10.3390/microorganisms8121873.

Bucardo F, Reyes Y, Becker-Dreps S, Bowman N, Gruber JF, Vinjé J, Espinoza F, Paniagua M, Balmaseda A, Svensson L, Nordgren J. 2017. Pediatric norovirus GII.4 infections in Nicaragua, 1999-2015. Infection, Genetics and Evolution 55:305-312. DOI: 10.1016/j.meegid.2017.10.001. Bucardo F. 2018. Understanding asymptomatic norovirus infections. EClinicalMedicine 2-3:7-8 DOI: 10.1016/j.eclinm.2018.09.005.

Peer) reviewing PDF | (2020:12:56205:2:0:NEW 17 Jun 2021) 
298 Chen MY, Chen WC, Chen PC, Hsu SW, Lo YC. 2016. An outbreak of norovirus gastroenteritis 299 associated with asymptomatic food handlers in Kinmen, Taiwan. BMC Public Health 16:372 300 DOI: 10.1186/s12889-016-3046-5.

301 Chhabra P, de Graaf M, Parra GI, Chan MC, Green K, Martella V, Wang Q, White PA, 302 Katayama K, Vennema H, Koopmans MPG, Vinjé J. 2019. Updated classification of norovirus 303 genogroups and genotypes. Journal of General Virology 100:1393-1406 DOI: 10.1099/jgv.0.001318.

Colston JM, Francois R, Pisanic N, Peñataro Yori P, McCormick BJJ, Olortegui MP, Gazi MA, Svensen E, Ahmed MMM, Mduma E, Liu J, Houpt ER, Klapheke R, Schwarz JW, Atmar RL, Black RE, Kosek MN. 2019. Effects of child and maternal histo-blood group antigen status on symptomatic and asymptomatic enteric infections in early childhood. The Journal of Infectious Diseases 220:151-162 DOI: 10.1093/infdis/jiz072.

Debbink K, Lindesmith LC, Donaldson EF, Baric RS. 2012. Norovirus immunity and the great escape. PLOS Pathogens 8:e1002921 DOI: 10.1371/journal.ppat.1002921.

de Graaf M, van Beek J, Koopmans MP. 2016. Human norovirus transmission and evolution in a 313 changing world. Nature Reviews Microbiology 14:421-33 DOI: 10.1038/nrmicro.2016.48. pattern and genotype distribution of norovirus infection in Japan. The Pediatric Infectious Diseases Journal 29:e32-34 DOI: 10.1097/INF.0b013e3181d742bf.

317 Fumian TM, Fioretti JM, Lun JH, Dos Santos IAL, White PA, Miagostovich MP. 2019.

318 Detection of norovirus epidemic genotypes in raw sewage using next generation sequencing. 319 Environment International 123:282-291 DOI: 10.1016/j.envint.2018.11.054. 
320 Franck KT, Lisby M, Fonager J, Schultz AC, Böttiger B, Villif A, Absalonsen H, Ethelberg S.

321 2015. Sources of calicivirus contamination in foodborne outbreaks in Denmark, 2005-2011-

322 The role of the asymptomatic food handler. The Journal of Infectious Diseases 211:563-70 DOI:

323 10.1093/infdis/jiu479.

324 Gaythorpe KAM, Trotter CL, Lopman B, Steele M, Conlan AJK. 2018. Norovirus transmission 325 dynamics: A modelling review. Epidemiology \& Infection 146:147-158 DOI:

$32610.1017 / \mathrm{S} 0950268817002692$.

327 Hall AJ, Lopman BA, Payne DC, Patel MM, Gastañaduy PA, Vinjé J, Parashar UD. 2013.

328 Norovirus disease in the United States. Emerging Infectious Diseases 19:1198-1205 DOI:

329 10.3201/eid1908.130465.

330 Jeong AY, Jeong HS, Lee JS, Park YC, Lee SH, Hwang IG, Kim YJ, Kim YJ, Jo MY, Jung S,

331 Kim K, Cheon DS. 2013. Occurrence of norovirus infections in asymptomatic food handlers in

332 South Korea. Journal of Clinical Microbiology 51:598-600 DOI: 10.1128/JCM.01856-12.

333 Kambhampati A, Koopmans M, Lopman BA. 2015. Burden of norovirus in healthcare facilities

334 and strategies for outbreak control. Journal of Hospital Infection 89:296-301 DOI:

$335 \quad 10.1016 /$ j.jhin.2015.01.011.

336 Kazama S, Masago Y, Tohma K, Souma N, Imagawa T, Suzuki A, Liu X, Saito M, Oshitani H,

337 Omura T. 2016. Temporal dynamics of norovirus determined through monitoring of municipal

338 wastewater by pyrosequencing and virological surveillance of gastroenteritis cases. Water

339 Research 92:244-253 DOI: 10.1016/j.watres.2015.10.024.

340 Kirkwood CD, Streitberg R. 2008. Calicivirus shedding in children after recovery from

341 diarrhoeal disease. Journal of Clinical Virology 43:346-348 DOI: 10.1016/j.jcv.2008.08.001. 
342 Lindesmith LC, Donaldson EF, Lobue AD, Cannon JL, Zheng DP, Vinje J, Baric RS. 2008.

343 Mechanisms of GII.4 norovirus persistence in human populations. PLOS Medicine 5:e31 DOI:

344 10.1371/journal.pmed.0050031.

345 Lopman B, Gastañaduy P, Park GW, Hall AJ, Parashar UD, Vinjé J. 2012. Environmental

346 transmission of norovirus gastroenteritis. Current Opinion in Virology 2:96-102 DOI:

347 10.1016/j.coviro.2011.11.005.

348 Matsuyama R, Miura F, Nishiura H. 2017. The transmissibility of noroviruses: Statistical

349 modeling of outbreak events with known route of transmission in Japan. PLOS One 12:e0173996

350 DOI: 10.1371/journal.pone.0173996.

351 Food Safety Bureau, Ministry of Health, Labour and Welfare of Japan. 2003. On the detection of

352 noroviruses.. Available from: https:/www.mhlw.go.jp/topics/syokuchu/kanren/kanshi/031105-

353 1.html (accessed on 2 March 2021).

354 Food Poisoning Subgroup, Food Hygiene Committee, Ministry of Health, Labour and Welfare of 355 Japan. 2007. Interventions against norovirus outbreaks (proposal). Available from:

356 https://www.mhlw.go.jp/shingi/2007/10/d1/s1012-5a.pdf (accessed on 2 March 2021).

357 Ministry of Health, Labour and Welfare of Japan. 2019. Revisions on Food Poisoning

358 Countermeasures and Survey Manual of Food Poisoning. Available from:

359 https://www.mhlw.go.jp/content/000496802.pdf (accessed on 2 March 2021).

360 Miura F, Matsuyama R, Nishiura H. 2018. Estimating the asymptomatic ratio of norovirus

361 infection during foodborne outbreaks with laboratory testing in Japan. Journal of Epidemiology

362 28:382-387 DOI: 10.2188/jea.JE20170040. 
363 Motomura K, Yokoyama M, Ode H, Nakamura H, Mori H, Kanda T, Oka T, Katayama K, Noda

364 M, Tanaka T, Takeda N, Sato H; Norovirus Surveillance Group of Japan. 2010. Divergent

365 evolution of norovirus GII/4 by genome recombination from May 2006 to February 2009 in

366 Japan. Journal of Virology 84:8085-8097 DOI: 10.1128/JVI.02125-09.

367 Newman KL, Moe CL, Kirby AE, Flanders WD, Parkos CA, Leon JS. 2016. Norovirus in

368 symptomatic and asymptomatic individuals: Cytokines and viral shedding. Clinical \&

369 Experiment Immunology 184:347-357 DOI: 10.1111/cei.12772.

370 Nicolay N, McDermott R, Kelly M, Gorby M, Prendergast T, Tuite G, Coughlan S, McKeown P,

371 Sayers G. 2011. Potential role of asymptomatic kitchen food handlers during a food-borne

372 outbreak of norovirus infection, Dublin, Ireland, March 2009. Eurosurveillance 16:19931.

373 O’Ryan M, Riera-Montes M, Lopman B. 2017. Norovirus in Latin America: Systematic review

374 and meta-analysis. The Pediatric Infectious Disease Journal 36:127-134 DOI:

375 10.1097/INF.0000000000001369.

376 Ozawa K, Oka T, Takeda N, Hansman GS. 2007. Norovirus infections in symptomatic and

377 asymptomatic food handlers in Japan. Journal of Clinical Microbiology 45:3996-4005 DOI:

378 10.1128/JCM.01516-07.

379 Parra GI, Squires RB, Karangwa CK, Johnson JA, Lepore CJ, Sosnovtsev SV, Green KY. 2017.

380 Static and evolving norovirus genotypes: Implications for epidemiology and immunity. PLOS

381 Pathogens 13:e1006136 DOI: 10.1371/journal.ppat.1006136.

382 Phattanawiboon B, Nonthabenjawan N, Boonyos P, Jetsukontorn C, Towayunanta W,

383 Chuntrakool K, Ngaopravet K, Ruchusatsawat K, Uppapong B, Sangkitporn S, Mekada E,

384 Matsuura Y, Tatsumi M, Mizushima H. 2020. Norovirus transmission mediated by

Peer] reviewing PDF | (2020:12:56205:2:0:NEW 17 Jun 2021) 
385 386

387 388

390

391

392

393

394

395

396

397

398

399

400

401

402

403

404

405

asymptomatic family members in households. PLOS One 15:e236502 DOI:

10.1371/journal.pone.0236502.

Phillips G, Tam CC, Rodrigues LC, Lopman B. 2010. Prevalence and characteristics of asymptomatic norovirus infection in the community in England. Epidemiology \& Infection 138:1454-1458 DOI: 10.1017/S0950268810000439.

Pringle K, Lopman B, Vega E, Vinje J, Parashar UD, Hall AJ. 2015. Noroviruses: Epidemiology, immunity and prospects for prevention. Future Microbiology 10:53-67 DOI:

10.2217/fmb.14.102. PMID: 25598337.

Qi L, Xiang X, Xiong Y, Ling H, Shen H, Deng W, Tang W, Shen T, Li Q. 2017. Outbreak of acute gastroenteritis caused by norovirus genogroup II attributed to contaminated cold dishes on a cruise ship in Chongqing, China, 2017. International Journal of Environmental Research and Public Health 15:2823 DOI: 10.3390/ijerph15122823.

Qi R, Huang YT, Liu JW, Sun Y, Sun XF, Han HJ, Qin XR, Zhao M, Wang LJ, Li W, Li JH, Chen C, Yu XJ. 2018. Global prevalence of asymptomatic norovirus infection: A meta-analysis. EClinicalMedicine 2-3:50-58 DOI: 10.1016/j.eclinm.2018.09.001.

Ramani S, Atmar RL, Estes MK. 2014. Epidemiology of human noroviruses and updates on vaccine development. Current Opinion in Gastroenterology 30:25-33 DOI:

10.1097/MOG.0000000000000022.

Rockx B, De Wit M, Vennema H, Vinjé J, De Bruin E, Van Duynhoven Y, Koopmans M. 2002. Natural history of human calicivirus infection: A prospective cohort study. Clinical Infectious Diseases 35:246-253 DOI:10.1086/341408. 
406 Rushton SP, Sanderson RA, Reid WDK, Shirley MDF, Harris JP, Hunter PR, O'Brien SJ. 2019.

407 Transmission routes of rare seasonal diseases: The case of norovirus infections. Philosophical

408 Transactions of the Royal Society of London B Biological Sciences 374:20180267 DOI:

$409 \quad 10.1098 /$ rstb.2018.0267.

410 Sabrià A, Pintó RM, Bosch A, Bartolomé R, Cornejo T, Torner N, Martínez A, Simón M,

411 Domínguez A, Guix S.. 2016. Norovirus shedding among food and healthcare workers exposed

412 to the virus in outbreak settings. Journal of Clinical Virology 82:119-125 DOI:

$413 \quad 10.1016 /$ j.jcv.2016.07.012.

414 Saito M, Goel-Apaza S, Espetia S, Velasquez D, Cabrera L, Loli S, Crabtree JE, Black RE,

415 Kosek M, Checkley W, Zimic M, Bern C, Cama V, Gilman RH; Norovirus Working Group in

416 Peru. 2014 Multiple norovirus infections in a birth cohort in a Peruvian Periurban community.

417 Clinical Infectious Diseases 58:483-491 DOI: 10.1093/cid/cit763.

418 Teunis PF, Moe CL, Liu P, Miller SE, Lindesmith L, Baric RS, Le Pendu J, Calderon RL. 2008.

419 Norwalk virus: How infectious is it? Journal of Medical Virology 80:1468-1476 DOI:

$420 \quad 10.1002 / j m v .21237$.

421 Teunis PF, Sukhrie FH, Vennema H, Bogerman J, Beersma MF, Koopmans MP. 2015. Shedding 422 of norovirus in symptomatic and asymptomatic infections. Epidemiology \& Infection 143:17104231717 DOI: 10.1017/S095026881400274X.

424 Tohma K, Lepore CJ, Gao Y, Ford-Siltz LA, Parra GI. 2019. Population genomics of GII.4

425 noroviruses reveal complex diversification and new antigenic sites involved in the emergence of 426 pandemic strains. mBio 10:e2202-19. DOI: 10.1128/mBio.02202-19. 
427 White PA. 2014. Evolution of norovirus. Clinical Microbiology and Infection 20:741-745 DOI:

$428 \quad 10.1111 / 1469-0691.12746$.

429

430 
431 Figure legends

432 Figure 1: Descriptive characteristics of norovirus outbreaks in Japan, 2005-2019.

433 A. Geographic distribution of outbreaks in seven regions. Numbers represent the total number of 434 documented outbreaks in 2005-2019 $(\mathrm{n}=1728)$. Geographic locations of Nara and Tokyo are 435 pointed by arrows. B. Long-term variations in numbers of outbreaks by year $(\mathrm{n}=1728)$. C.

436 Monthly variation in the numbers of outbreaks $(n=1728)$. D \& E. Types of outbreak setting 437 (e.g., facilities and schools). Only the data for Nara (D) and Tokyo (E) permitted examination of 438 the yearly numbers of outbreaks by type.

Figure 2: Temporal variations in Norovirus outbreak size in Japan, 2005-2019, estimated using a virus detection program $(n=434)$.

A. Seasonal variations in outbreak size. Summer is represented by May-October $(n=56)$ and winter by November-April $(\mathrm{n}=378)$. The median outbreak sizes in summer and winter were 15.5 and 16.0 cases, respectively. The maximum outbreak sizes in summer and winter were 99 and 308 cases, respectively. An F-test indicated that the winter outbreaks varied more than the summer outbreaks $(\mathrm{F}=2.6, \mathrm{p}<0.0001)$. B. Yearly variations in the numbers of confirmed cases in 2005-2019. In both A and B, the horizontal line represents the overall mean. The box extends from the lower to the upper quartile. Whiskers extend from the first quartile $-(1.5 \times$ interquartile range) to the third quartile $+(1.5 \times$ interquartile range $)$. 
Figure 1

Figure 1: Descriptive characteristics of norovirus outbreaks in Japan in 2005-2019.

A. Geographic distribution of outbreaks in seven regions. Numbers represent the total number of documented outbreaks in 2005-2019 $(n=1728)$. B. Long-term variations in numbers of outbreaks by year $(n=1728)$. C. Monthly variations in the numbers of outbreaks $(n=1728) . D \& E$. Types of outbreak setting (e.g., facilities and schools). Only the data for Nara (D) and Tokyo (E) allowed us to examine the yearly numbers of outbreaks by type. 


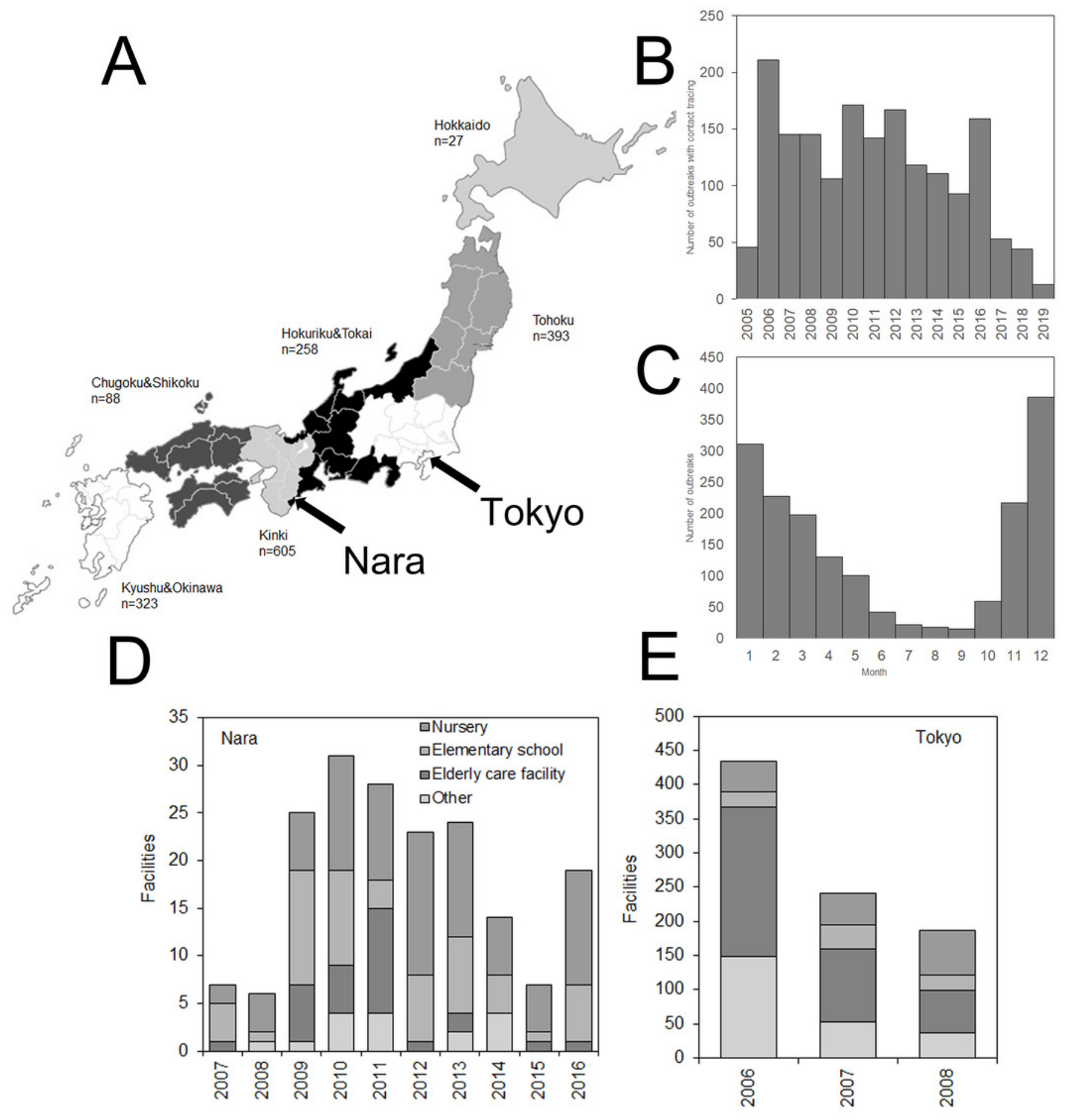




\section{Figure 2}

Figure 2: Temporal variations in Norovirus outbreak size in Japan in 2005-2019, estimated with a virus detection program $(n=434)$.

A. Seasonal variations in outbreak size. Summer is represented by May-October $(n=56)$ and winter by November-April $(n=378)$. The median outbreak sizes in summer and winter were 15.5 and 16.0, respectively. The maximum outbreak sizes in summer and winter were 99 and 308 patients, respectively, and an F-test indicated that the winter outbreaks varied more than the summer outbreaks $(F=2.6, p<0.0001)$. B. Yearly variations in the numbers of confirmed cases in 2005-2019. In both A and B, the horizontal straight line represents the grand mean. The box extends from the lower to upper quartile. Whiskers extend from the 1st quartile $-(1.5 \times$ interquartile range $)$ to the $3 r d$ quartile $+(1.5 \times$ interquartile range).
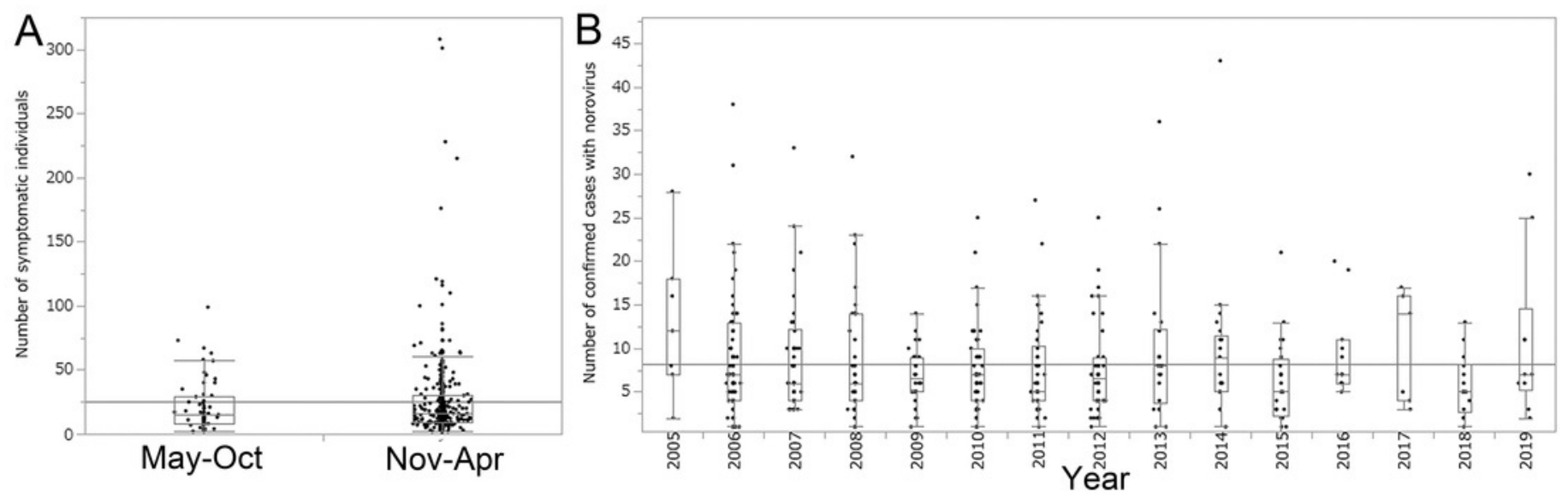


\section{Table $\mathbf{1}$ (on next page)}

Table 1: Probability of infection given exposure, the asymptomatic ratio, and the probability of virus detection estimated from outbreak data $(n=434)$ in 2005-2019, Japan.

Numbers in parentheses represent $95 \%$ confidence intervals. All three parameters were assumed to vary every year. 
1 Table 1: Probability of infection given exposure, the asymptomatic ratio, and the

2 probability of virus detection estimated from outbreak data $(n=434)$ in 2005-2019, Japan.

\begin{tabular}{|c|c|c|c|c|c|c|}
\hline \multirow{2}{*}{$\begin{array}{l}\text { Year } \\
2005\end{array}$} & \multicolumn{2}{|c|}{$\begin{array}{c}(p) \text { Risk of infection } \\
(\%)\end{array}$} & \multicolumn{2}{|c|}{$\begin{array}{c}\text { (s) Risk of } \\
\text { asymptomatic } \\
\text { infection (\%) }\end{array}$} & \multicolumn{2}{|c|}{$\begin{array}{c}\text { (q) Risk of virus- } \\
\text { positive outcome (\%) }\end{array}$} \\
\hline & 74.3 & $(67.2-80.7)$ & 4.4 & $(1.4-9.9)$ & 72.5 & $(64.1-80.0)$ \\
\hline 2006 & 64.7 & $(61.8-67.5)$ & 15.9 & $(13.2-18.9)$ & 79.8 & $(76.6-82.7)$ \\
\hline 2007 & 59.8 & $(56.0-63.5)$ & 25.5 & $(21.3-30.1)$ & 87.1 & $(83.1-90.4)$ \\
\hline 2008 & 68.9 & $(64.7-73.1)$ & 15.7 & (11.9-20.1) & 82.4 & (77.9-86.4) \\
\hline 2009 & 62.4 & $(57.3-67.4)$ & 17.2 & $(12.5-22.8)$ & 82.0 & $(76.3-86.8)$ \\
\hline 2010 & 65.6 & $(61.9-69.3)$ & 18.7 & $(15.1-22.7)$ & 88.0 & $(84.5-91.0)$ \\
\hline 2011 & 59.1 & $(55.2-62.9)$ & 19.1 & $(15.1-23.5)$ & 83.6 & $(79.4-87.4)$ \\
\hline 2012 & 61.4 & $(57.2-65.5)$ & 18.7 & $(14.5-23.3)$ & 83.6 & $(79.0-78.5)$ \\
\hline 2013 & 65.7 & $(60.5-70.6)$ & 18.4 & $(13.7-23.9)$ & 94.1 & $(90.2-96.9)$ \\
\hline 2014 & 59.0 & $(53.9-64.0)$ & 16.6 & $(11.9-22.1)$ & 91.1 & $(87.4-95.3)$ \\
\hline 2015 & 56.7 & $(50.8-62.6)$ & 25.3 & $(18.8-32.8)$ & 80.7 & $(73.5-86.8)$ \\
\hline 2016 & 71.8 & $(64.2-78.7)$ & 24.3 & $(16.8-33.0)$ & 92.0 & $(85.2-96.5)$ \\
\hline 2017 & 61.8 & $(53.2-70.1)$ & 21.3 & $(13.1-31.5)$ & 85.5 & $(76.0-92.5)$ \\
\hline 2018 & 68.3 & $(59.8-76.4)$ & 22.2 & $(14.1-32.1)$ & 81.8 & $(72.2-89.3)$ \\
\hline 2019 & 59.0 & $(51.9-65.9)$ & 15.7 & $(9.7-23.4)$ & 95.8 & $(90.5-98.7)$ \\
\hline
\end{tabular}


4 Numbers in parentheses represent 95\% confidence intervals. All three parameters were assumed

5 to vary every year.

6 


\section{Table 2 (on next page)}

Table 2: Probability of infection given exposure, the asymptomatic ratio, and the probability of virus detection estimated by genogroup and genotype based on outbreak data for 2005-2019, Japan.

Numbers in parentheses represent $95 \%$ confidence intervals. All three parameters were assumed to vary with genogroup or genotype. Groups were mutually exclusive (e.g., GI+GII includes outbreaks that involved both $\mathrm{Gl}$ and GII and were not counted as part of the GI group or GII group). Similarly, GII represents outbreaks caused by genogroup GII, excluding those genotyped as GII.4. Note that the GII group could still include GII.4 viruses that were not genotyped. 
1 Table 2: Probability of infection given exposure, the asymptomatic ratio, and the

2 probability of virus detection estimated by genogroup and genotype based on outbreak

3 data for 2005-2019, Japan.

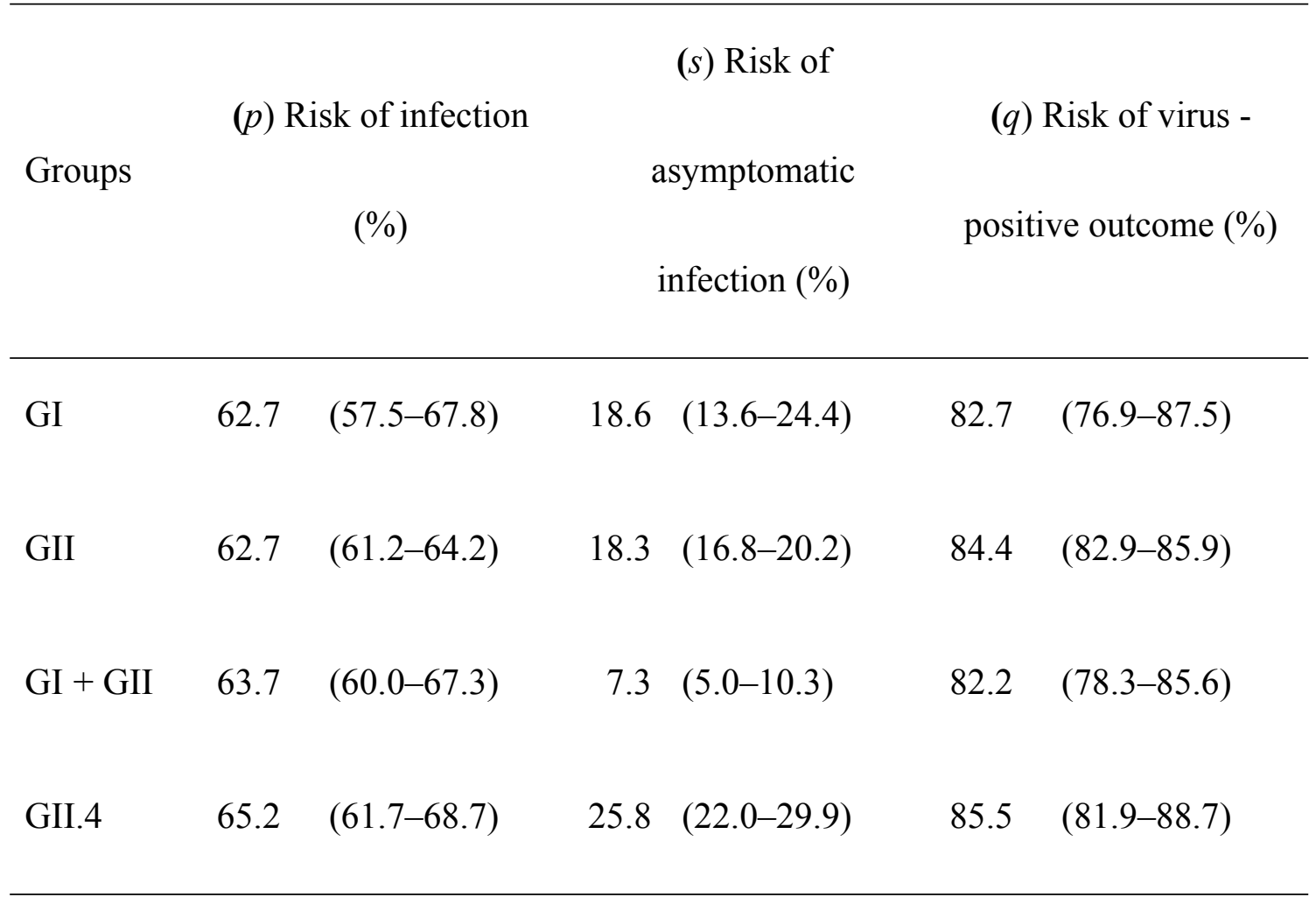

4 Numbers in parentheses represent $95 \%$ confidence intervals. All three parameters were assumed

5 to vary with genogroup or genotype. Groups were mutually exclusive (e.g., GI+GII includes

6 outbreaks that involved both GI and GII detected and were not counted as part of the GI group or

7 GII group). Similarly, GII represents outbreaks caused by genogroup GII, excluding those

8 genotyped as GII.4. Note that the GII group could still include GII.4 viruses that were not

9 genotyped. 
